\title{
Prevention of Hyperactive Gag Reflex during Dental Procedures in COVID-19 Pandemic
}

\author{
Kaveh Nasiri ${ }^{10} \quad$ Aleksandra Dimitrova ${ }^{2(0)}$ \\ ${ }^{1}$ Independent Researcher, Essen, Germany \\ 2 Department of Hematology, Internal Oncology \& Stem Cell \\ Transplant, Evang. Hospital Essen-Werden, Essen, Germany \\ Eur Dent Res Biomater J 2021;2:52-54.
}

Address for correspondence Kaveh Nasiri, DDS, MSc, Essen 45138 , Germany (e-mail: DDS.Nasiri@web.de).
Severe acute respiratory syndrome coronavirus type 2 (SARS-CoV-2) was detected in December 2019 in China, and since then the COVID-19 pandemic has significantly affected our lives. Toward the end of 2020, several vaccines were developed and manufactured to protect individuals and prevent the spread of COVID-19. In addition to vaccination, medical masks, social distancing, and hand disinfection can also help to reduce the risk of transmission. One of the routes through which SARS-CoV-2 can be transmitted is saliva, despite having an antiviral effect against infectious diseases. In this pandemic, any dental procedures that cause irritation and secretion of salivary glands, the spread of saliva, and oral or nasopharyngeal discharges should be considered as a potential transmission risk factor of COVID-19. ${ }^{1-3}$ This brief letter focuses on the concept of the gag reflex and its control during dental treatment in the current pandemic.

Because gag reflex is a serious obstacle for performing various dental procedures, it is a major concern for the clinician. Gag reflex or laryngospasm is the contraction of the throat which is caused when the clinician touches the oral cavity. There are five sensitive areas in the oral cavity known as trigger zones, namely the base of the tongue, fauces, palate, uvula, and posterior pharyngeal. Gagging can be classified into somatic and psychogenic. Somatic gagging can be caused by touching the oral cavity, particularly trigger zones; however, other factors, including smell and sound of dental instruments or devices may cause psychogenic gagging. Moreover, hypersalivation which is caused by oromotor function, dysphagia, decreased central control, and coordination can lead to gaging., ${ }^{4,5}$ Gagging is stressful for the patient and clinician during dental procedures, specifically during the current pandemic. The results of one study in which vomiting was stimulated in vitro to measure the spread of secretions through UV lighting showed that vomit particles would be expelled to 3.1 $\mathrm{m} \times 2.6 \mathrm{~m}$, contaminating an area of $7.8 \mathrm{~m}^{2}{ }^{6}$ This means that gagging is a potential risk factor in transmitting COVID19 and hence managing gag reflex has a high priority, particularly among patients suspected to have COVID-19.

There are many ways to avoid or reduce gag reflex: (1) acupuncture techniques i.e., inserting needles into CV 24 and PC 6 acupuncture points, (2) therapy on P6 acupoint via lowlevel laser, (3) acupressure, i.e., pressuring a point without piercing the skin during dental procedures, (4) hypnosis, (5) trans-cutaneous electrical nerve stimulation, (6) psychological therapy, (7) earplug technique, (8) pharmacological treatment, including glycopyrrolate fluid solution (sialanar), sedatives (nitrous oxide), and local and general anesthetics, (9) miscellaneous techniques, i.e., various treatment modifications that clinicians find effective, such as using rubber dam and sectional impression tray, (10) relaxation techniques; passive techniques, such as listening to music, using dark glasses, and avoiding seeing dental instruments and active techniques, for example breathing through the nose or relaxing abdominal breathing, (11) distraction techniques, such as asking the patient to focus on keeping their feet away from the dental unite, putting salt on the tongue, closing eyes, and rinsing the mouth with ice cold water, and (12) desensitization techniques, including brushing or touching the soft palate and tongue directly with finger. ${ }^{4,7}$

Silva et al reported that in patients with severe gag reflex, the extraoral periapical X-ray reduced the gag reflex compared with conventional intraoral X-ray during root canal treatment. A panoramic radiograph or cone-beam computed tomography (CBCT) can also be used as an alternative examination. ${ }^{8}$ In another recent review study, Nasiri and Wrbas stated that electronic apex locators, regardless of their generation, prove to be beneficial for patients suffering from gag reflex or those who cannot endure X-ray film or sensor. ${ }^{9}$ published online

February 16, 2022
DOI https://doi.org/ $10.1055 / \mathrm{s}-0041-1741526$. ISSN 2791-7452.
(C) 2022. The Author(s).

This is an open access article published by Thieme under the terms of the Creative Commons Attribution License, permitting unrestricted use, distribution, and reproduction so long as the original work is properly cited. (https://creativecommons.org/licenses/by/4.0/)

Thieme Medical and Scientific Publishers Pvt. Ltd., A-12, 2nd Floor, Sector 2, Noida-201301 UP, India 
Another challenge for clinicians in this pandemic is the cyclic vomiting syndrome (CVS); it was first recognized in pediatrics; however, it is now confirmed that adults can also suffer from CVS. The exact etiology of CVS is unknown; nonetheless, stress, anxiety, and lack of sleep are all linked to CVS, which causes severe nausea and vomiting for hours. While there is not an absolute cure for CVS, using intravenous fluids and nausea medications, such as ondansetron or prochlorperazine, can be helpful. Moreover, sedation medications, such as lorazepam or diphenhydramine are effective, as well. A calm and dim environment can considerably help to reduce stress in the case of CVS and is thus recommended. ${ }^{10}$ In light of the information in this brief letter, the clinician can play a significant role in managing of patients with a gag reflex and reducing the spread of COVID-19.

\section{Conflict of Interest}

None declared.

\section{References}

1 Nasiri K. COVID-19 and the antiviral effect of saliva. Eur J Dent 2020;14(S01)S177-S178
2 Nasiri K, Dimitrova A, Nasiri K. Does the use of mouthwash before collecting saliva specimen increase the possibility of a falsenegative result in COVID-19 testing? Dent Oral Maxillofac Res 2020;6:1-2

3 Nasiri K, Dimitrova A. Current state of developed COVID-19 vaccines; light at the end of the tunnel. Int J Endovasc Treat Innov Tech 2021;2(01):17-24.

4 Forbes-Haley C, Blewitt I, Puryer J. Dental management of the 'gagging' patient-an update. Int J Dent Health Sci 2016;3(02):423-431

5 Steffen A, Jost W, Bäumer T, et al. Hypersalivation: update of the German S2k guideline (AWMF) in short form. J Neural Transm (Vienna) 2019;126(07):853-862

6 Makison Booth C. Vomiting Larry: a simulated vomiting system for assessing environmental contamination from projectile vomiting related to norovirus infection. J Infect Prev 2014;15(05):176-180

7 Anand MV, Rai R, Bettie NF, Ramachandiran HSolomon Praveena $S$. Acupuncture - an effective tool in the management of gag reflex. J Pharm Bioallied Sci 2015;7(Suppl 2):S677-S679

8 E Silva MH, Coelho MS, Santos MF, de Lima CO, Campos CN. The use of an alternative extraoral periapical technique for patients with severe gag reflex. Case Rep Dent 2016;2016:3206845

9 Nasiri K, Wrbas KT. Accuracy of different generations of apex locators in determining working length; a systematic review and meta-analysis. Saudi Dent J 2022;34(01):11-20

10 Davis A, Nichols CJ, Bryant JH. Cyclic Vomiting Syndrome. In: StatPearls. Treasure Island (FL): StatPearls Publishing LLC; 2021 
